\title{
BLADDER DYSFUNCTION IN HEREDITARY SPASTIC PARAPLEGIA: WHAT TO EXPECT?
}

\section{Corresponding author}

Mark Braschinsky MD

neurologist,

Department of Neurology, University of Tartu,

Puusepa 2, Tartu, 51014, Estonia

e-mail: mark.braschinsky@kliinikum.ee

Tel: +3727318512

Fax: +3727318509

\section{Authors:}

Mark Braschinsky, MD

University of Tartu, Department of Neurology, Tartu, Estonia

Inga Zopp, RN

West Tallinn Central Hospital, Centre for Neurological Diseases, Tallinn, Estonia

Mart Kals, MSc

Department of Mathematics, University of Tartu, Tartu, Estonia

Sulev Haldre, MD, PhD

University of Tartu, Department of Neurology, Tartu, Estonia

Katrin Gross-Paju, MD, PhD

West Tallinn Central Hospital, Centre for Neurological Diseases, Tallinn, Estonia

Keywords: hereditary spastic paraplegia, neurogenic urinary bladder dysfunction, Strümpell-

Lorrain disease

Word count of text: 2262

Word count of the abstract: 246 


\section{ABSTRACT}

Background: Hereditary spastic paraplegia (HSP) comprises a group of rare neurodegenerative disorders characterized by progressive spasticity and hyperreflexia of the legs. Neurogenic bladder dysfunction is a well-recognized problem in patients with HSP, but it has not yet been described systematically in the literature. The aim of this study was to provide an evidential overview of the ways in which urinary dysfunction presents in HSP. Methods: Forty-nine patients with HSP were included and underwent evaluation. A history was followed by a semi-structured interview and, in those patients who consented, measurement of residual volume of urine (PVR) and urodynamic evaluation.

Results: Thirty-eight subjects $(77.6 \%)$ reported some type of urinary symptom. Subjective complaints of bladder problems showed a correlation with verified urinary dysfunction. There were no significant differences in the occurrence of urinary disturbances between the pure and complex forms of HSP (pHSP and cHSP, respectively). The most frequent symptoms were incontinence (69.4\%), hesitancy (59.2\%), increased frequency of micturition (55.1\%), and urgency (51.0\%). Incomplete bladder emptying was the rarest (36.7\%). The most common combination of symptoms was to have all of them (14.3\%). Incomplete bladder emptying as a complaint was associated with an increased risk of PVR. Women had a higher risk of increased voiding frequency.

Conclusions: To our knowledge, this work is the first systematic and disease-oriented overview of neurogenic bladder disturbances in patients with HSP. Our results may be useful to the clinicians who work with HSP patients, allowing them to make appropriate screening and management decisions. 


\section{INTRODUCTION}

Hereditary spastic paraplegia (HSP) is a group of rare neurodegenerative disorders characterized by progressive spasticity and hyperreflexia of the lower limbs. HSP has marked clinical and genetic heterogeneity. Although it is often referred to as Strümpell-Lorrain disease, it has been suggested that the term hereditary spastic paraparesis is more appropriate.[1] The main anatomo-pathological hallmark of HSP is retrograde degeneration of the cortico-spinal tracts and of the posterior columns. The disorder is classified clinically into "pure" (pHSP) and "complex" (cHSP) forms. Patients with the pHSP form present with spasticity and motor deficit in the legs, brisk reflexes, and Babinski's sign, often accompanied by deep sensory impairment and sphincter disturbances. The cHSP form is associated with a number of other neurological or extra-neurological features, such as ataxia, distal amyotrophy, cognitive impairment, optic neuropathy, and retinopathy, among others.[2] Although, neurogenic bladder dysfunctions, which are those that result from interference with the normal nerve pathways associated with urination, are a well-recognized problem in patients with HSP, they have not yet been described systematically in the literature. A PubMed search using the terms "HSP" and "voiding" currently returns two publications; "HSP" and "sphincter" return eight; "HSP" and "urinary" return 12 ; and "HSP" and "bladder" return nine. Overall, this yields a total of 22 publications, the earliest dated 1973.[3-24] A number of these are review articles that describe HSP in general, but do not focus on bladder dysfunction.

The aim of this study was to provide an evidential overview of urinary dysfunction presentations in HSP.

\section{METHODS}


This study was approved by Ethics Review Committee on Human Research of the University of Tartu.

Patients who had been diagnosed with HSP, as defined by the diagnostic criteria described by Fink et al. and summarized by Reid, were invited to participate in the study.[2526] Contact information was acquired from an epidemiological study performed in Estonia.[27] Forty-nine of the 59 Estonian patients with HSP who were invited (30 men and 19 women) agreed to participate in this study and gave written, informed consent. Of these, $41(84 \%)$ were diagnosed with pHSP and $8(16 \%)$ with cHSP. Nine participants (18\%) had mutations in the SPG4 gene. The mean age of the participants was 50.9 years, ranging between 11 and 75 years. The mean disease duration was 20.2 years, and ranged from 3 to 42 years.

All subjects were questioned in general about both distressing and more benign problems with their bladder function. Distressing problems were defined as those causing a major impact on lifestyle. This history was followed by a semi-structured interview conducted by the qualified nurse continence advisor. She specifically inquired as to urinary frequency, urgency, hesitancy, incomplete bladder emptying, and incontinence. Patients were asked whether they had a history of urinary tract infections. After the interview, all subjects were evaluated for residual volume of urine (PVR) and urinalysis. Frequency of micturition was considered to be elevated if it exceeded 8 times in 24 hours and the patient had less than 6 hours of uninterrupted sleep.

PVR was measured by BladderScan (model BVI 2500, DxU Diagnostic Ultrasound Corporation). Clinically relevant incomplete emptying was defined as PVR greater than 100 $\mathrm{ml}$, measured immediately after voiding. For urodynamic evaluation, the consenting patients were divided into two groups depending upon whether or not they had PVR. 
Forty-five of the 49 patients agreed to be evaluated for spasticity and mobility. Modified Ashworth scale (MAS) and measurement of walking speed were used for the evaluation (the detailed results are described elsewhere).[28]

\section{Statistical analysis}

Frequencies of the study variables were determined. The Fisher's exact test or the Chi-square test were used to assess the associations. A Spearman's rank correlation analysis was applied to investigate the effects of MAS on complaints of urinary dysfunction and PVR. Results are presented by odds ratios (OR) with $95 \%$ confidence interval $(95 \% \mathrm{CI})$ or correlation coefficients (CC). Free software R (version 2.2) was used for all statistical analysis. A $p$ value less than 0.05 was defined as statistically significant.

\section{RESULTS}

Of the 49 participants, 38 (77.6\%) spontaneously complained of at least one urinary symptom. There was no statistically significant difference between patients with or without mutations in the SPG4 gene $(p=0.40)$. The following symptoms were reported: frequency (20 patients); urgency (19); incontinence (16); hesitancy (12); and incomplete emptying (12), showing no correlation with the presence or absence of the mutations in the SPG4 gene (with $p$ value ranging from 0.4545 to 1.0$)$. Distressing symptoms were reported by 21 patients, and non-distressing symptoms by 18 . There was no statistically significant difference between patients with or without mutations in the SPG4 gene $(p=0.6339)$. The presence of complaints was not influenced by neither the degree of spasticity $(p=0.936)$ nor the walking speed $(p=$ 0.100). 
During the semi-structured interview, the following problems were identified:

incontinence (34 patients, $69.4 \%)$; hesitancy $(29,59.2 \%)$; increased frequency of micturition (27, 55.1\%); urgency $(25,51.0 \%)$; and incomplete bladder emptying $(18,36.7 \%)$ (table 1$)$.

Table 1. The occurrence of different types of urinary dysfunction

\begin{tabular}{|c|c|c|c|c|c|}
\hline \multicolumn{2}{|c|}{ Type of dysfunction } & \multirow{2}{*}{ N (\%) } \\
\cline { 1 - 4 } FR & HE & \multirow{2}{*}{ IN } & UR & \multirow{2}{*}{ IE } & \\
\hline+ & + & + & + & + & $7(14.3)$ \\
\hline+ & + & + & - & + & $6(12.2)$ \\
\hline+ & - & + & + & - & $5(10.2)$ \\
\hline+ & + & + & + & - & $4(8.2)$ \\
\hline- & + & + & + & - & $2(4.1)$ \\
\hline- & + & + & - & - & $2(4.1)$ \\
\hline- & + & - & + & - & $2(4.1)$ \\
\hline- & + & - & - & - & $2(4.1)$ \\
\hline- & + & + & - & + & $2(4.1)$ \\
\hline- & - & + & + & - & $2(4.1)$ \\
\hline- & - & + & - & + & $1(2.0)$ \\
\hline- & + & - & + & + & $1(2.0)$ \\
\hline+ & + & + & - & - & $1(2.0)$ \\
\hline+ & - & + & - & - & $1(2.0)$ \\
\hline+ & - & + & - & + & $1(2.0)$ \\
\hline+ & - & - & + & - & $1(2.0)$ \\
\hline+ & - & - & - & - & $1(2.0)$ \\
\hline- & - & - & + & - & $1(2.0)$ \\
\hline- & - & - & - & - & $7(14.3)$ \\
\hline & & & TOTAL & $49(100)$ \\
\hline
\end{tabular}

$\mathrm{FR}=$ frequency; $\mathrm{HE}=$ hesitancy; IN = incontinence; $\mathrm{UR}=$ urgency; IE = incomplete emptying; $\mathrm{N}$ = number of subjects. 
Seven patients (14.3\%) had all of the aforementioned complaints (frequency, hesitancy, urgency, incontinence and incomplete emptying). Isolated mild hesitancy was revealed in two men, and isolated urgency by one woman, who had no spontaneous complaints and whose PVR was within normal limits. All other subjects without complaints tested normal during the interview. Different combinations of the various subtypes of urinary dysfunction were present in all subjects with non-distressing symptoms $(n=18)$. All patients complaining of distressing urinary problems had incontinence, with only 2 denying an increased frequency of urination. The presence of complaints showed a positive correlation with verified urinary dysfunction (table 2).

Table 2. The frequency and correlation between subjective and actual urinary dysfunction. Non-distressing complaints were defined as those that do not compel the patients to make changes in their everyday activities. Significance was defined as $p<0.05$.

\begin{tabular}{|r|c|c|}
\hline \multirow{2}{*}{ Complaints of urinary dysfunction } & \multicolumn{2}{|c|}{ Actual urinary dysfunction } \\
\cline { 2 - 3 } & Yes & No \\
\hline No complaints & $3(7 \%)$ & $7(100 \%)$ \\
Non-distressing problems & $18(43 \%)$ & $0(0 \%)$ \\
Distressing problems & $21(55 \%)$ & $0(0 \%)$ \\
\hline Total & $42(100 \%)$ & $7(100 \%)$ \\
\hline p-value $^{*}$ & \multicolumn{2}{|c|}{$<001$} \\
\hline
\end{tabular}

*Fisher's exact test.

Women had a higher risk of increased voiding frequency, with an OR of 5.625 (95\% $\mathrm{CI}=1.498-21.118, p=0.0105)$. Otherwise, neither age, gender, nor disease duration were significant risk factors for any type of bladder disturbances in HSP (table 3). Twenty-one patients $(42.9 \%)$ had a history of urinary tract infection. 
Table 3. Correlation between age and disease duration in subjects with different type of urinary disturbances.

\begin{tabular}{|l|c|c|c|c|c|c|}
\hline \multicolumn{2}{|c|}{} & Frequency & Hesitancy & Urgency & Incontinence & PVR \\
\hline Age & $\begin{array}{c}\text { Odds ratio } \\
(95 \% \mathrm{CI})\end{array}$ & $\begin{array}{c}1.025 \\
(0.985-1.067)\end{array}$ & $\begin{array}{c}1.030 \\
(0.989-1.073)\end{array}$ & $\begin{array}{c}0.982 \\
(0.943-1.022)\end{array}$ & $\begin{array}{c}1.034 \\
(0.991-1.078)\end{array}$ & $\begin{array}{c}1.022 \\
(0.978-1.067)\end{array}$ \\
\cline { 2 - 7 } & $p$-value* & 0.228 & 0.158 & 0.364 & 0.125 & 0.336 \\
\hline \multirow{2}{*}{$\begin{array}{l}\text { Disease } \\
\text { duration }\end{array}$} & $\begin{array}{c}\text { Odds ratio } \\
(95 \% \mathrm{CI})\end{array}$ & 1.012 & 1.053 & 1.021 & 1.026 & 1.017 \\
& $(0.959-1.067)$ & $(0.994-1.115)$ & $(0.968-1.077)$ & $(0.968-1.088)$ & $(0.963-1.075)$ \\
\cline { 2 - 7 } & $p$-value $^{*}$ & 0.674 & 0.081 & 0.454 & 0.393 & 0.539 \\
\hline
\end{tabular}

$\mathrm{PVR}=$ residual volume of urine; $\mathrm{CI}=95 \%$ confidence interval; $*$ Chi-square test.

PVR was measured in all subjects. It was greater than $100 \mathrm{ml}$ (range: $212-477 \mathrm{ml}$ ) in 5 men and 1 woman. The presence of a PVR over $100 \mathrm{ml}$ correlated negatively with walking speed $(\mathrm{CC}=-0.438 ; p=0.003)$ and positively with the degree of spasticity in legs as measured at different levels, including hip abduction $(\mathrm{CC}=0.398 ; p=0.007)$. The complaint of incomplete bladder emptying showed a statistically significant correlation with an increased risk of the PVR exceeding $100 \mathrm{ml}(\mathrm{OR}=2.426 ; 95 \% \mathrm{CI}=1.104-5.331 ; p=0.027)$. The presence of a PVR over $100 \mathrm{ml}$ tended to be a risk factor for urinary infection $(\mathrm{OR}=5.2$; $95 \% \mathrm{CI}=0.929-29.095)$, although it did not reach the level of statistical significance $(p=$ 0.0606). Less than $100 \mathrm{ml}$ (range: $5-73 \mathrm{ml}$ ) was detected in another 24 subjects.

On urodynamic evaluation, two groups, consisting of 4 consenting patients each who either did or did not have PVR, were compared. Three out of 4 patients with PVR showed dyssynergy and were unable to void independently. Dyssynergy was noted in only one patient without PVR, whose voiding was independent. Three of 6 patients with more than $100 \mathrm{ml}$ of PVR were currently performing clean intermittent self-catheterisation (CIC). Two of 6 had performed CIC in the past but had discontinued it for personal reasons. One of 6 patients had never performed CIC. 
Seventeen of 49 patients used oxybutynine, 11 regularly and 6 intermittently. Thirteen of 27 patients with subjective complaints of frequency and 7 of the 25 who complained of urgency used oxybutynine. All 17 subjects who used oxybutynine complained of continuing incontinence.

There were no statistically significant differences in the occurrence of urinary tract disturbances between pHSP and cHSP forms (78 and 75\%, respectively).

\section{DISCUSSION}

The published literature contains a number of reports, descriptive or interventional, concerning the relationship between HSP and voiding.[4] Absent, however, has been an overview encompassing the occurrence, type, and severity of neurogenic bladder dysfunctions in HSP and of sufficient scope allow the disease to be evaluated as a distinct nosologic unit without further sub-classification. In this study, we have demonstrated that symptoms related to bladder disturbance are common in HSP, with up to $78 \%$ of patients reporting some kind of urinary dysfunction. This suggests that a substantial proportion of HSP patients are at risk for neurogenic voiding problems. When a neurological condition affects the function of bladder, the urinary symptoms can take different forms, including urgent, frequent, or hesitant voiding, incontinence, or partial emptying. Each of these neurourological symptoms was present in some proportion of the HSP patients studied here. The most frequent complaints were incontinence and hesitancy of voiding, which should therefore be assessed in any clinical evaluation of HSP patients. The most prevalent combination of symptoms, reported by $15 \%$ of subjects, was the entire set of complaints (table 1). Hence, to ensure that patients with HSP receive the appropriate treatment, care should be taken to thoroughly assess all potential urinary complaints. 
In this study, the presence of the complaints usually indicated urinary dysfunction that could be verified. Conversely, our results suggest that it is highly improbable that asymptomatic HSP patients have verifiable urinary dysfunction (table 2). This may provide a useful guide in clinical practice to identify, based on history, those HSP patients needing more extensive investigation of bladder dysfunction. Other clinical clues are the degree of spasticity and the walking speed, which are the clinical hallmarks of disability in HSP. We found, that both parameters could be used as predictors of neurourological disturbances - the higher the spasticity and the lower the mobility are, the higher risk of bladder dysfunction is.

It is well documented that incomplete bladder emptying is a significant risk factor for symptomatic urinary tract infections and upper urinary tract complications. Fortunately, this neuro-urological problem is relatively easy to manage: PVR volume greater then $100 \mathrm{ml}$ requires CIC. [29] Consequently, it is important to identify patients who may require the procedure. In the current study, some patients who did complain about incomplete bladder emptying had an increased PVR volume, including values exceeding $100 \mathrm{ml}(\mathrm{OR}=2.4)$. Those with PVR had a higher incidence of dyssynergy on urodynamic evaluation, when compared with HSP patients without PVR (3/4 and 1/4, respectively). Unfortunately, in our study the total number of patients who agreed to participate in urodynamic evaluation was too small either to perform adequate statistical analysis or to draw meaningful conclusions. However, the clinical relevance of this possible trend is clear, as the simultaneous contraction of the sphincter and the detrusor can result in high intravesicular pressure, potentially endangering the upper urinary tract.[30] Interestingly, our results indicated that the percentage of HSP patients who had elevated PVR was relatively low, at approximately $12 \%$. Hence, close observation of these at-risk patients is crucial so that timely implementation of appropriate treatment can be used to avoid possible serious complications. 
PVR values over $100 \mathrm{ml}$ were also associated with an increased risk of symptomatic lower urinary tract infection $(\mathrm{OR}=5.2)$. However, in our study this correlation was a trend that failed to reach statistical significance, unlike the more definitive results that have been reported for studies of CNS disorders like multiple sclerosis (MS), spinal cord lesions, and others, where the relationship between PVR and lower urinary tract infection is wellestablished.[31] This might be explained by the nature of the disease, since HSP affects the pyramidal tract, and thus spares the sensory feedback from the bladder. In MS and in most of other spinal lesions, the afferent impulses from the bladder are usually impaired, which may be related to the fact that, in those cases, the patient only becomes aware of the residual volume at higher values.

The absence of an observed correlation between bladder dysfunction and disease duration may be explained by the typically benign course of HSP. The differences in age and gender upon clinical presentation, including the extent of urinary disturbances, are potentially related to different genetic forms of the disease. However, it is controversial due to a great variability of the disorder with the same genetic basis (including intrafamilial variations).[32]

Our results further indicated that both the pHSP and cHSP clinical forms of the disease are associated with a similar incidence of urinary dysfunction (78 and 75\%, respectively). In terms of prevalence, character and severity of neurourological complaints we did not find any differences between patients with or without mutations in the SPG4 gene. Although some studies have suggested that the clinical and genetic forms of HSP differ in the prevalence of bladder dysfunction, this is still under debate, and any differences may simply be related to the extent of pyramidal involvement.[1] The same conclusion was drawn from the studies of other neurological conditions, such as MS, that similarly affect pyramidal pathways and produce urinary symptoms.[33] 
This study has some limitations. Despite a substantial total number of participants, some subgroups were too small for firm conclusions to be drawn from the observed trends, highlighting the need for further investigation in this area. In addition, this descriptive study depended on the patients' own reports, which are by their nature subjective. Nevertheless, all efforts were made to reduce any possible biases.

To conclude, this work is, to our knowledge, the first systematic and disease-oriented overview of neurogenic bladder disturbances in patients with HSP. HSP is a relatively rare neurodegenerative disorder, making it difficult to study many of its manifestations, including bladder disturbances. Nonetheless, we suggest that all attempts should be made to quantify the presence of urinary symptoms in HSP. The results may help to guide the clinicians who treat HSP patients to select the appropriate screening and management protocols. 


\section{Acknowledgements}

This study is supported by Estonian Science Foundation research grant nr. ETF5680.

\section{Funding}

This study is funded by Estonian Science Foundation (Endla 4, Tallinn 10142, Estonia) research grant nr. ETF5680.

\section{Competing interests}

None.

\section{Ethics approval}

This study was approved by Ethics Review Committee on Human Research of the University of Tartu (Ülikooli 18-304, Tartu 50090, Estonia).

The Corresponding Author has the right to grant on behalf of all authors and does grant on behalf of all authors, an exclusive licence (or non exclusive for government employees) on a worldwide basis to the BMJ Publishing Group Ltd and its Licensees to permit this article (if accepted) to be published in the Journal of Neurology, Neurosurgery \& Psychiatry editions and any other BMJPGL products to exploit all subsidiary rights, as set out in our licence (http://jnnp .bmjjournals.com/ifora/licence.pdf). 


\section{References}

1. Tallaksen CM, Dürr A, Brice A. Recent advances in hereditary spastic paraplegia. Curr Opin in Neurol 2001;14(4):457-463.

2. McDermott CJ, White K, Bushby K, et al. Hereditary spastic paraparesis: a review of new developments. J Neurol Neurosurg Psychiatry 2000;69:150-160.

3. Bertelli M, Cecchin S, Lorusso L, et al. Identification of a novel mutation in the spastin gene (SPG4) in an Italian family with hereditary spastic paresis. Panminerva Med 2006;48(3):193-197.

4. Bushman W, Steers WD, Meythaler JM. Voiding dysfunction in patients with spastic paraplegia: urodynamic evaluation and response to continuous intrathecal baclofen. Neurourol Urodyn 1993;12(2):163-170.

5. Cartlidge NE, Bone G. Sphincter involvement in hereditary spastic paraplegia. Neurology 1973;23(11):1160-1163.

6. Colazza GB, Di Gennaro G, Quarato PP, et al. A case of a rare association of spastic paraplegia and type III syndactyly. Eur J Neurol 2002;9(1):105-107.

7. Dürr A, Camuzat A, Colin E, et al. Atlastin1 mutations are frequent in young-onset autosomal dominant spastic paraplegia. Arch Neurol 2004;61(12):1867-1872.

8. Efstratiadis G, Memmos D, Tsiaousis G, et al. Strumpell's disease in a family with hereditary focal segmental glomerulosclerosis. Ren Fail 2006;28(4):351-354.

9. Fink JK. Hereditary spastic paraplegia. Curr Neurol Neurosci Rep 2006;6(1):65-76.

10. Harding AE. Hereditary "pure" spastic paraplegia: a clinical and genetic study of 22 families. J Neurol Neurosurg Psychiatry 1981;44(10):871-883.

11. Heinzlef O, Paternotte C, Mahieux F, et al. Mapping of a complicated familial spastic paraplegia to locus SPG4 on chromosome 2p. J Med Genet 1998;35(2):89-93. 
12. Jennum P, Neerup Jensen L, Fenger K, et al. Motor evoked potentials from the external anal sphincter in patients with autosomal dominant pure spastic paraplegia linked to chromosome 2p. J Neurol Neurosurg Psychiatry 2001;71(4):561-562.

13. Ki CS, Lee WY, Han DH, et al. A novel missense mutation (I344K) in the SPG4gene in a Korean family with autosomal-dominant hereditary spastic paraplegia. J Hum Genet 2002;47(9):473-477.

14. Matsuura T, Sasaki H, Wakisaka A, et al. Autosomal dominant spastic paraplegia linked to chromosome 2p: clinical and genetic studies of a large Japanese pedigree. $J$ Neurol Sci 1997;151(1):65-70.

15. Meierkord H, Nürnberg P, Mainz A, et al. 'Complicated' autosomal dominant familial spastic paraplegia is genetically distinct from 'pure' forms. Arch Neurol 1997;54(4):379-384.

16. Meijer IA, Dupré N, Brais B, et al. SPG4 founder effect in French Canadians with hereditary spastic paraplegia. Can J Neurol Sci 2007;34(2):211-214.

17. Naidu S, Dlouhy SR, Geraghty MT, et al. A male child with the rumpshaker mutation, $\mathrm{X}$-linked spastic paraplegia/Pelizaeus-Merzbacher disease and lysinuria. J Inherit Metab Dis 1997;20(6):811-816.

18. Opjordsmoen S, Nyberg-Hansen R. Hereditary spastic paraplegia with neurogenic bladder disturbances and syndactylia. Acta Neurol Scand 1980;61(1):35-41.

19. Saltuari L, Kronenberg M, Marosi MJ, et al. Long-term intrathecal baclofen treatment in supraspinal spasticity. Acta Neurol (Napoli) 1992;14(3):195-207.

20. Scheltens P, Bruyn RP, Hazenberg GJ. A Dutch family with autosomal dominant pure spastic paraparesis (Strümpell's disease). Acta Neurol Scand 1990;82(3):169-173. 
21. Topaloğlu H, Pinarli G, Erdem H, et al. Clinical observations in autosomal recessive spastic paraplegia in childhood and further evidence for genetic heterogeneity. Neuropediatrics 1998;29(4):189-194.

22. Valente EM, Brancati F, Caputo V, et al. Novel locus for autosomal dominant pure hereditary spastic paraplegia (SPG19) maps to chromosome 9q33-q34. Ann Neurol 2002;51(6):681-685.

23. Webb S, Patterson V, Hutchinson M. Two families with autosomal recessive spastic paraplegia, pigmented maculopathy, and dementia. J Neurol Neurosurg Psychiatry 1997;63(5):628-632.

24. Woods G, Black G, Norbury G. Male neonatal death and progressive weakness and immune deficiency in females: an unknown X linked condition. J Med Genet 1995;32(3):191-196.

25. Fink JK, Heiman-Patterson T, Bird T et al. Hereditary Spastic Paraplegia: Advances in Genetic Research. Neurology 1996;46(6):1507-1514.

26. Reid, E. Pure hereditary spastic paraplegia. J Med Genet 1997;34(6):499-503.

27. Braschinsky M, Lüüs S-M, Gross-Paju K, et al. The prevalence of hereditary spastic paraplegia and the occurrence of SPG4 mutations in Estonia. Neuroepidemiology 2009;32:89-93.

28. Braschinsky M, Parts K, Maamägi H, et al. Functional assesment of lower extremities in hereditary spastic paraplegia. Arch Phys Med Rehabil In press.

29. Fowler CJ, O’Malley KJ. Investigation and management of neurogenic bladder dysfunction. J Neurol Neurosurg Psychiatry 2003;74 Suppl 4:27-31.

30. Fowler CJ. Integrated control of lower urinary tract - clinical perspective. $\mathrm{Br} J$ Pharmacol 2006;147 Suppl 2:S14-24. 
31. Foxman B. Epidemiology of urinary tract infections: incidence, morbidity, and economic costs. Am J Med 2002;113(1):5-13.

32. Orlacchio A, Kawarai T, Totaro A, et al. Hereditary spastic paraplegia: clinical genetic study of 15 families. Arch Neurol 2004;61(6):849-855.

33. Fowler CJ, Kalsi V. Bladder dysfunction in multiple sclerosis. Neurol Sci 2006;27(4):323-327. 